\title{
The non-structural protein $\mu$ NS of piscine orthoreovirus (PRV) forms viral factory-like structures
}

\author{
Hanne Merethe Haatveit ${ }^{1}$ (D), Ingvild B. Nyman' ${ }^{1}$, Turhan Markussen ${ }^{1,2}$, Øystein Wessel ${ }^{1}$, Maria Krudtaa Dahle ${ }^{3}$ \\ and Espen Rimstad ${ }^{1 *}$
}

\begin{abstract}
Piscine orthoreovirus (PRV) is associated with heart- and skeletal muscle inflammation in farmed Atlantic salmon. The virus is ubiquitous and found in both farmed and wild salmonid fish. It belongs to the family Reoviridae, closely related to the genus Orthoreovirus. The PRV genome comprises ten double-stranded RNA segments encoding at least eight structural and two non-structural proteins. Erythrocytes are the major target cells for PRV. Infected erythrocytes contain globular inclusions resembling viral factories; the putative site of viral replication. For the mammalian reovirus (MRV), the non-structural protein $\mu N S$ is the primary organizer in factory formation. The analogous PRV protein was the focus of the present study. The subcellular location of PRV $\mu N S$ and its co-localization with the PRV $\sigma N S, \mu 2$ and $\lambda 1$ proteins was investigated. We demonstrated that PRV $\mu$ NS forms dense globular cytoplasmic inclusions in transfected fish cells, resembling the viral factories of MRV. In co-transfection experiments with $\mu N S$, the $\sigma N S, \mu 2$ and $\lambda 1$ proteins were recruited to the globular structures. The ability of $\mu$ NS to recruit other PRV proteins into globular inclusions indicates that it is the main viral protein involved in viral factory formation and pivotal in early steps of viral assembly.
\end{abstract}

\section{Introduction}

Piscine orthoreovirus (PRV) is a member of the family Reoviridae. The virus is associated with heart and skeletal muscle inflammation (HSMI), an important emerging disease in the intensive farming of Atlantic salmon (Salmo salar) [1, 2]. HSMI is mainly observed during the seawater grow-out phase and there is often a prolonged disease development [3]. The cumulative mortality varies from negligible to $20 \%$, while the morbidity is almost $100 \%$ in affected cages [3]. PRV seems to be ubiquitous in Norwegian salmon farms [4]. Fish kept at high stocking density with frequent handling experience a stressful environment that may result in immunosuppression and a greater disease burden, thus facilitating the rapid spread of pathogens [5]. PRV has also been detected in

\footnotetext{
*Correspondence: espen.rimstad@nmbu.no

${ }^{1}$ Department of Food Safety and Infectious Biology, Faculty of Veterinary Medicine and Biosciences, Norwegian University of Life Sciences, Postboks 8146 Dep, 0033 Oslo, Norway

Full list of author information is available at the end of the article
}

wild salmon, but no lesions consistent with HSMI have been discovered in the wild population [6].

Phylogenetic analysis indicates that PRV branches off the common root of the genera Orthoreovirus and Aquareovirus, but most closely related to the orthoreoviruses [7, 8]. PRV differs from other orthoreoviruses like mammalian reoviruses (MRVs) and avian reoviruses (ARVs) in the ability to infect salmonid fish species at low temperatures, and in the preference for erythrocytes as one of the main target cells. The genome of PRV comprises ten double-stranded RNA (dsRNA) segments distributed in the classical orthoreoviral groups of three large, three medium and four small segments $[1,8,9]$. Currently, the PRV genome has been found to encode at least ten primary translation products. However, there is only a limited number of functional studies concerning the different proteins expressed by this virus $[10,11]$. Based upon sequence homology to MRV, and the presence of conserved structures and motifs, eight of the deduced translation products are assumed structural components forming the orthoreovirus particle with an 
inner core and an outer capsid, while two of the translation products are non-structural proteins $[8,12]$.

A common feature for the non-structural proteins of reoviruses is their ability to form viral factories [13, 14]. Viral factories, also known as viroplasms or viral replication centers, are intracellular compartments for replication, packaging and assembly of viral particles [13, 15]. Several RNA and DNA viruses have been reported to induce these specialized membranous compartments within the cytoplasm of infected cells [16-18]. They commonly form as invaginations in a variety of organelles such as mitochondria, endoplasmic reticulum, lysosomes, peroxisomes, Golgi apparatus or chloroplasts $[18,19]$. The factory scaffold facilitates spatial coordination of viral genome replication and assembly with the use of cell resources [18]. The viral factory inclusions seen during MRV infection consist of viral dsRNA, viral proteins, partially and fully assembled viral particles, microtubules and thinner filaments suggested to be intermediate structures [20]. Although organization of viral factories varies between different virus families, several fundamental similarities exist. Viruses utilize cellular biosynthetic pathways for their morphogenesis and propagation, and use a variety of mechanisms to avoid being wiped out by the cellular antiviral response [13, 21]. In the viral factories the viral pathogen-associated molecular patterns are shielded from inducing the activation of cellular innate responses [19].

Erythrocytes are major target cells for PRV, and in infected erythrocytes globular inclusions are formed and contain both PRV protein and dsRNA [22, 23]. The inclusions resemble the globular viral factories seen in MRV type 3 Dearing (T3D) prototype strain infected cells $[19,22]$. Furthermore, the PRV inclusions contain reovirus-like particles as observed by transmission electron microscopy (TEM) [22]. This suggests that PRV, like MRV, forms viral factories in infected cells.

MRV $\mu$ NS is the scaffolding protein that organizes viral factories during MRV infection [24]. Comparison of the PRV $\mu$ NS amino acid sequence with the homologous proteins from MRV and ARV has revealed a very low sequence identity of only $17 \%$, however, partially conserved motifs are present [8]. The latter includes a C-terminal motif shown for MRV $\mu \mathrm{NS}$ to be required for the recruitment of clathrin to viral factories $[8,25]$. Furthermore, predictions of MRV and ARV $\mu$ NS show two $\alpha$-helical coils in their $\mathrm{C}$-terminal region required for inclusion formation [26-29]. A high $\alpha$-helical content in the C-terminal region is also predicted for the PRV $\mu$ NS, but coiled coil motifs are predicted with significantly lower probability than for MRV and ARV [8]. In addition, MRV and ARV have both been shown to produce two protein products from gene segment M3 [8,
30]. Whereas $\mu \mathrm{NS}$ represents the full-length isoform, a second in-frame AUG $\left(\mathrm{Met}_{41}\right)$ in the MRV protein represents the translational start site for the second isoform $\mu N S C$. In the ARV protein, post-translational cleavage near the $\mathrm{N}$-terminal region creates $\mu \mathrm{NSN}[8,30]$. In PRV $\mathrm{M} 3$, only one open reading frame (ORF) has been identified encoding the $\mu$ NS protein [8].

We hypothesized that the $\mu$ NS of PRV is an organization center in the assembly of progeny virus particles. The aim in this study was to examine the localization of PRV $\mu$ NS and its ability to interact with other PRV proteins in transfected cells.

\section{Materials and methods \\ Cells}

EPC cells (ATCC CRL-2872, Epithelioma papulosum cyprini) and CHSE-214 cells (ATCC CRL-1681, Chinook salmon embryo) were cultivated in Leibovitz-15 medium (L15, Life Technologies, Paisley, Scotland, UK) supplemented with $10 \%$ heat inactivated fetal bovine serum (FBS, Life technologies), $2 \mathrm{mM}$ L-glutamine, $0.04 \mathrm{mM}$ mercaptoethanol and $0.05 \mathrm{mg} / \mathrm{mL}$ gentamycin-sulphate (Life Technologies).

\section{Computer analyses}

Multiple sequence alignments were performed using AlignX (Vector NTI Advance ${ }^{\mathrm{TM}}$ 11, Invitrogen, Carlsbad, CA, USA) and protein secondary structure predictions using PSIPRED v3.0. The presence of putative nuclear localization signals (NLS) in PRV $\mu 2$ was investigated using PSORTII, PredictProtein [31] and NLS mapper. The GenBank accession numbers for the PRV $\mu$ NS, $\sigma \mathrm{NS}, \lambda 1$ and $\mu 2$ coding sequences of the present study are KR337478, KR337481, KR337475 and KR337476, respectively.

\section{Plasmid constructs}

Total RNA was isolated from homogenized tissue from a natural outbreak of HSMI in Atlantic salmon (MH050607) as previously described [8]. RNA was denatured at $95{ }^{\circ} \mathrm{C}$ for $5 \mathrm{~min}$ and transcribed into cDNA using SuperScript ${ }^{\circledR}$ III Reverse Transcriptase (RT) (Invitrogen) and Random Primers (Invitrogen) according to the manufacturer's protocol. PfuUltra II Fusion HS DNA polymerase (Agilent, Santa Clara, CA, USA) was used to amplify the ORFs of $\mu \mathrm{NS}, \sigma \mathrm{NS}, \mu 2$ and $\lambda 1$. The primers contained the sequences encoding flag-tag, myc-tag or HA-tag for protein recognition by antibodies [32]. Primer sequences are shown in Table 1. For both the full-length $\mu \mathrm{NS}$ and $\sigma \mathrm{NS}$ constructs, a pair of expression vectors was made encoding proteins tagged in either the C-terminus or the N-terminus; pcDNA3.1- $\mu$ NS-N-FLAG, pcDNA3.1- $\mu$ NS-C-FLAG, pcDNA3.1- $\sigma \mathrm{NS}-\mathrm{N}-\mathrm{MYC}$ and 
Table 1 Expression plasmids.

\begin{tabular}{|c|c|c|}
\hline Plasmid name & Primer & Sequence $\left(5^{\prime} \rightarrow 3^{\prime}\right)$ \\
\hline \multirow[t]{2}{*}{ pcDNA3.1- $\mu N S-N-F L A G$} & Forward & GCCGCTCGAGTCTAGAGCCACCATGGACTACAAAGACGATGACGACAAGATGGCTGAATCAATTACTTTTGG \\
\hline & Reverse & AAACGGGCCCTCTAGATCAGCCACGTAGCACATTATTCAC \\
\hline \multirow[t]{2}{*}{ pcDNA3.1- $\mu N S-C-F L A G$} & Forward & GCCGCTCGAGTCTAGAGCCACCATGCGCAAGCTGGACTTGGTTGCA \\
\hline & Reverse & AAACGGGCCCTCTAGATCACTTGTCGTCATCGTCTTTGTAGTCGCCACGTAGCACATTATTCACGCC \\
\hline \multirow[t]{2}{*}{ pcDNA3.1-๔NS-N-MYC } & Forward & GCCGCTCGAGTCTAGAGCCACCATGGAACAAAAACTCATCTCAGAAGAGGATCTGATGTCGAACTTTGATCTTGG \\
\hline & Reverse & AAACGGGCCCTCTAGACTAACAAAACATGGCCATGA \\
\hline \multirow[t]{2}{*}{ pCDNA3.1-øNS-C-MYC } & Forward & GCCGCTCGAGTCTAGAGCCACCATGTCGAACTTTGATCTTGG \\
\hline & Reverse & AAACGGGCCCTCTAGACTACAGATCCTCTTCTGAGATGAGTTTTTGTTCACAAAACATGGCCATGATGC \\
\hline \multirow[t]{2}{*}{ pcDNA3.1- $\mu 2-C-H A$} & Forward & GGCGGCCGCTCGAGTCTAGAATGCCTATCATAAACCTGCC \\
\hline & Reverse & GTTTAAACGGGCCCTCTAGAAGCGTAATCTGGAACATCGTATGGGTACTCACCAGCTGTAGACCACC \\
\hline \multirow[t]{2}{*}{ pcDNA3.1- $\lambda 1-N-H A$} & Forward & CGCTCGAGTCTAGAGCCACCATGTACCCATACGATGTTCCAGATTACGCTATGGAGCGACTTAAGAGGAAAG \\
\hline & Reverse & AAACGGGCCCTCTAGATTAGTTGAGTACAGGATGAG \\
\hline \multirow[t]{2}{*}{ pcDNA3.1- $\mu N S \triangle 743-753$} & Forward & GCCGCTCGAGTCTAGAGCCACCATGGACTACAAAGACGATGACGACAAGATGGCTGAATCAATTACTTTTTG \\
\hline & Reverse & AAACGGGCCCTCTAGATCACCAGTCATCTGAGCCACCAAA \\
\hline \multirow[t]{2}{*}{ pcDNA3.1- $\mu N S \triangle 736-752$} & Forward & GCCGCTCGAGTCTAGAGCCACCATGGACTACAAAGACGATGACGACAAGATGGCTGAATCAATTACTTTTTG \\
\hline & Reverse & AAACGGGCCCTCTAGATCAGTCGATGATTTTTGGAAACTC \\
\hline \multirow[t]{2}{*}{ pcDNA3.1- $\mu N S \triangle 1-401$} & Forward & GCCGCTCGAGTCTAGAGCCACCATGCCAACCACCTGGTATTCAAC \\
\hline & Reverse & AAACGGGCCCTCTAGATCACTTGTCGTCATCGTCTTTGTAGTCGCCACGTAGCACATTATTCACGCC \\
\hline \multirow[t]{2}{*}{ pcDNA3.1- $\mu N S \triangle 402-752$} & Forward & GCCGCTCGAGTCTAGAGCCACCATGGACTACAAAGACGATGACGACAAGATGGCTGAATCAATTACTTTTTG \\
\hline & Reverse & AAACGGGCCCTCTAGATCATGTGGTCAGGGAATAGTGCAT \\
\hline
\end{tabular}

Primers used in generating the constructs encoding PRV $\mu N S$ (M3), $\sigma N S(S 3), \mu 2$ (M1) and $\lambda 1$ (L3) and truncated versions of $\mu N S$.

Start codons are marked in bold and epitope tags in italic.

pcDNA3.1- $\sigma$ NS-C-MYC. For $\mu 2$, the tag was added only C-terminally and for $\lambda 1$ only N-terminally, pcDNA3.1$\mu 2-\mathrm{C}-\mathrm{MYC}$ and pcDNA3.1- $\lambda 1-\mathrm{N}-\mathrm{HA}$, respectively. Four truncated forms of the $\mu$ NS protein with flag-tags $\mathrm{C}$ - or $\mathrm{N}$-terminally depending on the truncation were also generated to determine sequence regions in PRV $\mu \mathrm{NS}$ involved in formation of viral factories during infection, pcDNA3.1- $\mu \mathrm{NS} \Delta 1-401$, pcDNA3.1- $\mu \mathrm{NS} \Delta 402-752$, pcDNA3.1- $\mu N S \Delta 736-752$ and pcDNA3.1- $\mu N S \Delta 743-752$ (Figure 1). In-fusion HD Cloning Kit (Clontech Laboratories, Mountain View, CA, USA) was used to clone PCR products into the $\mathrm{Xbal}$ restriction site of the eukaryotic expression vector pcDNA3.1(+) (Invitrogen). Sanger sequencing (GATC Biotech AG, Konstanz, Germany) verified all sequences. A pcDNA3.1 construct expressing the protein encoded by infectious salmon anemia virus (ISAV) segment 8 open reading frame 2 (S8ORF2) protein [33] was used as a control during transfections, immunoprecipitation and western blotting.

\section{Transfections of fish cells}

EPC and CHSE cells were seeded on gelatin embedded cover slips $(12 \mathrm{~mm})$ with pre-equilibrated L-15 growth medium at a density of $1.5 \times 10^{4}$ cells in a 24-well plate $24 \mathrm{~h}$ prior to transfection. Plasmids were transfected using Lipofectamine LTX reagent (Life Technologies)

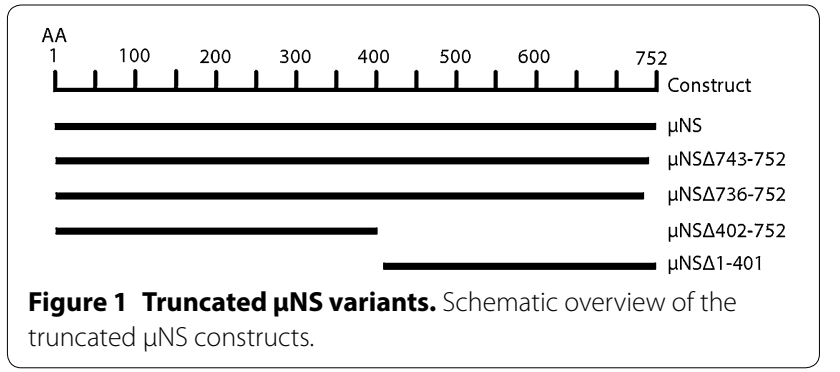

according to the manufacturer's instructions. In brief, 2 $\mu \mathrm{L}$ lipofectamine was mixed with $0.5 \mu \mathrm{g}$ plasmid and 0.5 $\mu \mathrm{L}$ PLUS reagent, and diluted in a total of $100 \mu \mathrm{L}$ OptiMEM (Life Technologies). After 5 min of incubation, the mixture was added to the cells and incubated at $20^{\circ} \mathrm{C}$ for $48 \mathrm{~h}$. When co-transfections were performed, a total of $0.4 \mu \mathrm{g}$ of each plasmid were used and the amount of PLUS reagent was increased to $0.8 \mu \mathrm{L}$.

\section{Immunofluorescence microscopy}

Transfected EPC and CHSE cells were fixed and stained using an intracellular Fixation and Permeabilization Buffer (eBioscience, San Diego, CA, USA). The cells were washed in Dulbecco's PBS (DPBS) with sodium azide. Intracellular fixation buffer was added before incubation 
with primary (1:1000) and secondary antibodies (1:400) diluted in permeabilization buffer according to the manufacturer's protocol. Antibodies against flag (mouse antiflag antibody) and HA (rabbit anti-HA antibody) were obtained from Sigma-Aldrich (St Louis, MO, USA), while antibodies against the myc epitope (goat anti-myc antibody) was obtained from Abcam (Cambridge, UK). Secondary antibodies against mouse immunoglobulin G (IgG), goat IgG and rabbit IgG were conjugated with either Alexa Fluor 488 or 594 obtained from Molecular Probes (Life Technologies). Hoechst trihydrochloride trihydrate (Life Technologies) was used for nuclear staining. The cover slips were mounted onto glass slides using Fluoroshield (Sigma-Aldrich) and prepared for microscopy as described above. Images were captured on an inverted fluorescence microscope (Olympus IX81) and on a confocal laser scanning microscope (Zeiss LSM 710).

\section{Immunoprecipitation}

A total of 5 million EPC cells were pelleted by centrifugation, resuspended in $100 \mu \mathrm{L}$ Ingenio Electroporation Solution (Mirus, Madison, WI, USA) and co-transfected with $8 \mu \mathrm{g}$ plasmid using the Amaxa T-20 program. pcDNA3.1$\mu N S-N-F L A G$ was co-transfected with pcDNA3.1- $\sigma$ NSN-MYC, pcDNA3.1- $\mu 2-\mathrm{C}-\mathrm{HA}$, pcDNA3.1- $\lambda 1-\mathrm{N}-\mathrm{HA}$ and pcDNA3.1 S8ORF2 (negative control) separately, using three parallel preparations. The transfected cells were transferred to $75 \mathrm{~cm}^{2}$ culture flasks containing $20 \mathrm{~mL}$ pre-equilibrated L-15 growth medium (described above). From each culture flask, $0.5 \mathrm{~mL}$ transfected cells were transferred to a 24-well plate intended for expression analysis by immunofluorescence microscopy. Cells were collected from the culture flasks $72 \mathrm{~h}$ post transfection (hpt), centrifuged at $5000 \mathrm{~g}$ for $5 \mathrm{~min}$ and resuspended in $1 \mathrm{~mL}$ Nonidet-P40 lysis buffer (1\% NP-40, $50 \mathrm{mM}$ Tris$\mathrm{HCl} \mathrm{pH} 8.0,150 \mathrm{mM} \mathrm{NaCl}, 2 \mathrm{mM}$ EDTA) containing Complete ultra mini protease inhibitor cocktail (Roche, Mannheim, Germany). The mix was incubated on ice for $30 \mathrm{~min}$, and then centrifuged at $9700 \mathrm{~g}$ for $12 \mathrm{~min}$ at $4{ }^{\circ} \mathrm{C}$. The supernatant was transferred to a new tube, added antibodies against the desired epitope tag or anti-S8ORF2 and incubated overnight at $4{ }^{\circ} \mathrm{C}$ with rotation. The Immunoprecipitation Kit Dynabeads Protein G (Novex, Life Technologies) was used for protein extraction and the beads prepared according to the manufacturer's protocol. The cell-lysate-antibody mixture was mixed with the protein $\mathrm{G}$ coated beads and incubated $2 \mathrm{~h}$ at $4{ }^{\circ} \mathrm{C}$. The beadsantibody-protein complex was washed according to the manufacturer's protocol.

\section{Western blotting}

The beads-antibody-protein complex was diluted in Sample Buffer (Bio-Rad, Hercules, CA, USA) and
Reducing Agent (Bio-Rad), denatured for 5 min at $95{ }^{\circ} \mathrm{C}$ and run in sodium dodecyl sulfate-polyacrylamide gel electrophoresis (SDS-PAGE), using 4-12\% Bis-Tris Criterion XT gel (Bio-Rad). Lysates from non-transfected EPC cells were used as a negative control, and Precision Plus Protein Western C Standards (Bio-Rad) as a molecular size marker. Following SDS-PAGE, the proteins were blotted onto a polyvinylidene fluoride (PVDF) membrane (Bio-Rad) and incubated with primary antibody (anti-flag 1:1000) at $4{ }^{\circ} \mathrm{C}$ overnight. After incubation with secondary antibody (Anti-mouse IgG-HRP, GE Healthcare, Buchinghamshire, UK), the proteins were detected by chemiluminescense using Amersham ECL Prime Western Blotting Detection Reagent (GE Healthcare).

\section{Results}

\section{Prediction of secondary structure}

The predicted secondary structure profiles of PRV and MRV $\mu$ NS were similar despite low sequence identity (Figure 2). The PRV $\mu$ NS sequence in this study differs by twenty-three nucleotides of which twenty are silent (not shown) to that analyzed in a previous study (GU994018) [8]. The three amino acids that differed between the two PRV $\mu$ NS sequences did not cause significant changes to the predicted secondary structures as determined by the PSIPRED program. The remaining three nucleotides all result in synonymous amino acid differences, i.e., displaying similar physiochemical properties $\left(\mathrm{M} / \mathrm{L}_{94}, \mathrm{I} / \mathrm{V}_{451}\right.$ and $\left.\mathrm{A} / \mathrm{V}_{498}\right)$. For $\sigma \mathrm{NS}$, the difference is six nucleotides and for $\lambda 1$ twenty-eight, all silent. For $\mu 2$, the difference is fifteen nucleotides, all silent except for one synonymous substitution $\left(\mathrm{R} / \mathrm{K}_{113}\right)$.

\section{$\mu N S$ forms viral factory-like structures}

EPC cells transfected with pcDNA3.1- $\mu \mathrm{NS}-\mathrm{N}-\mathrm{FLAG}$ 48 hpt showed small, dense globular inclusions evenly distributed in the cytoplasm with some larger perinuclear inclusions $48 \mathrm{hpt}$ (Figure 3A). A similar staining pattern was seen with the corresponding C-terminally flag-labelled construct (Figure 3A, insert), and in CHSE cells (not shown). EPC cells transfected with the oNS-NMYC, $\mu 2-\mathrm{C}-\mathrm{HA}$ or $\lambda 1-\mathrm{N}-\mathrm{HA}$ constructs were also examined 48 hpt (Figure $3 \mathrm{~B}-\mathrm{D}$ ). The $\sigma \mathrm{NS}-\mathrm{N}-\mathrm{MYC}$ protein was evenly distributed in the cytoplasm possibly with some minor nuclear localization (Figure 3B). A nucleocytoplasmic distribution pattern was also observed with the C-terminally myc-labelled $\sigma \mathrm{NS}$ (Figure 3B, insert). Both the $\mu 2-\mathrm{C}-\mathrm{HA}$ and $\lambda 1-\mathrm{N}-\mathrm{HA}$ proteins were evenly distributed in the cytoplasm (Figure $3 \mathrm{C}$ and D), with the former showing minor staining in the nucleus of some cells (not shown). Non-transfected cells did not show any staining (not shown). 


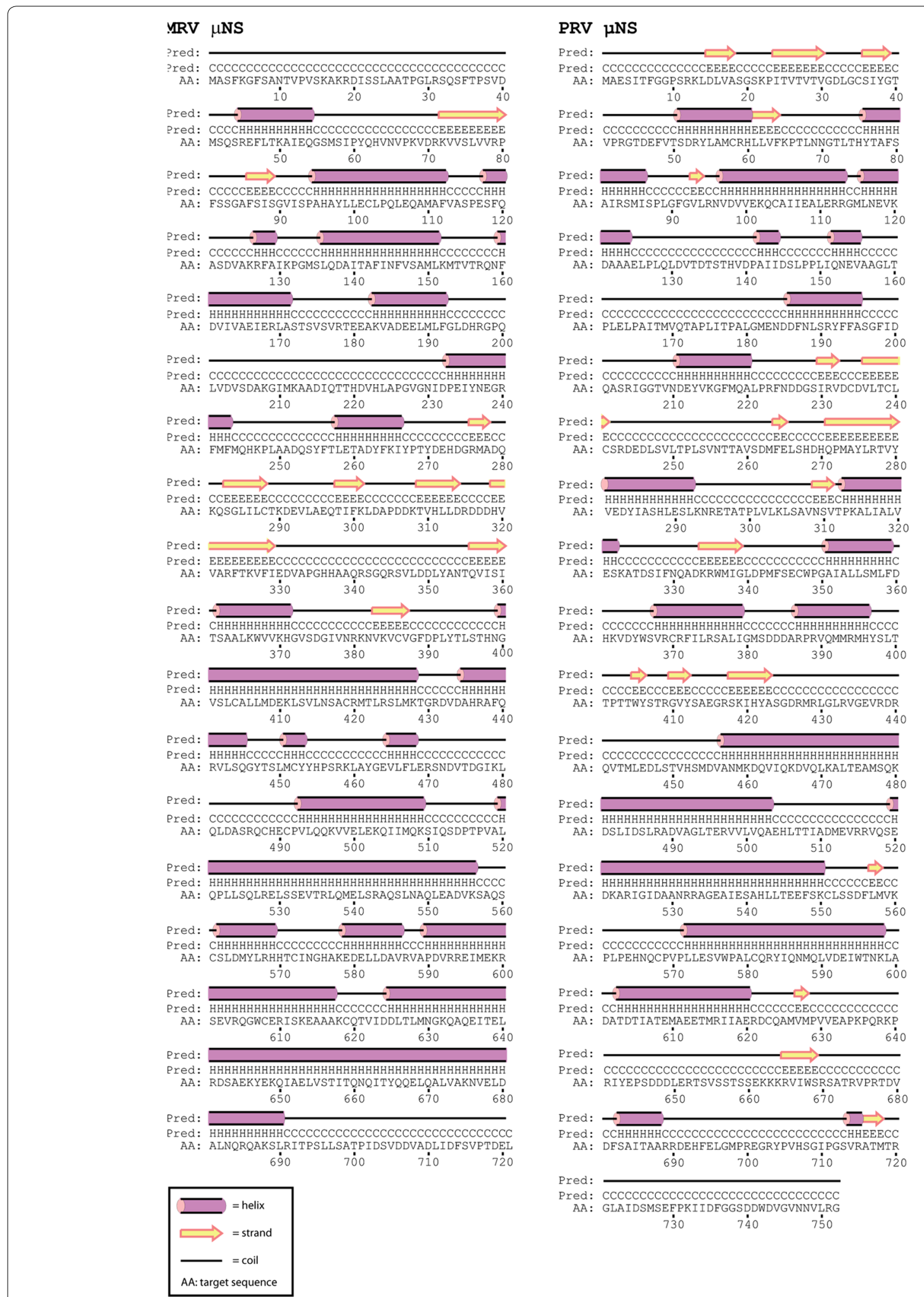

Figure 2 Secondary structure predictions. Secondary structure predictions of the $\mu N S$ proteins from PRV and MRV (PSIPRED). Accession numbers for the MRV and PRV proteins are NC004281 and KR337478, respectively. 

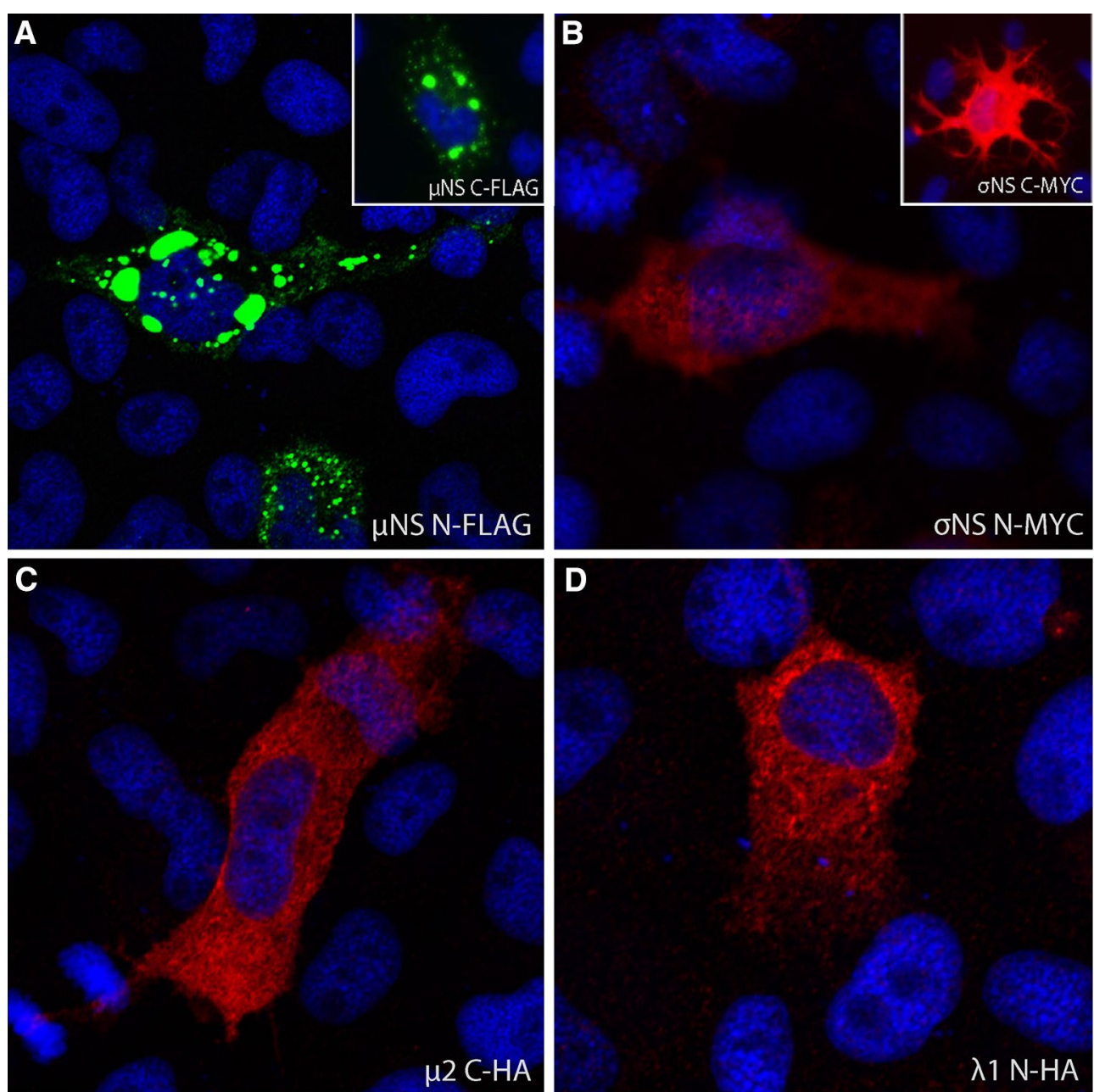

Figure 3 Subcellular localization of PRV proteins. EPC cells transfected with four different PRV plasmid constructs $(\mu N S, \sigma N S, \lambda 1, \mu 2)$ processed for fluorescence microscopy $48 \mathrm{hpt}$. A EPC cells expressing $\mu \mathrm{NS} N$-FLAG. Boxed region in top left corner shows EPC cells expressing $\mu N S-C-F L A G$. B EPC cells expressing $\sigma N S$ N-MYC. Boxed region shows $\sigma N S-C-M Y C$. C EPC cells expressing $\mu 2-C-H A$. D EPC cells expressing $\lambda 1-N-H A$.

$\sigma N S, \lambda 1$ and $\mu 2$ are recruited to viral factory-like structures Viral proteins interacting with $\mu \mathrm{NS}$ were identified by co-transfecting EPC cells with pcDNA3.1- $\mu$ NS-NFLAG and separately with each of the $\sigma \mathrm{NS}-\mathrm{N}-\mathrm{MYC}$, $\mu 2-\mathrm{C}-\mathrm{HA}$ or $\lambda 1-\mathrm{N}-\mathrm{HA}$ constructs. The $\mu \mathrm{NS}$ protein retained its globular distribution pattern in the presence of the other PRV proteins $48 \mathrm{hpt}$ (Figure 4). In contrast, the staining pattern for $\sigma \mathrm{NS}, \mu 2$ and $\lambda 1$ proteins changed from an evenly cytoplasmic distribution to globular inclusions co-localizing wholly or partially with the $\mu \mathrm{NS}$ protein (Figure $4 \mathrm{~A}-\mathrm{C}$ ). Co-localization with $\mu$ NS was most pronounced for $\sigma \mathrm{NS}$, and $\sigma \mathrm{NS}$ was no longer found in the nucleus (Figure 4A). For $\mu 2$, the change in distribution was not as pronounced as for $\sigma \mathrm{NS}$ and $\lambda 1$, but in some cells $\mu 2$ formed small punctuated structures partially overlapping with the $\mu \mathrm{NS}$ globular inclusions (Figure 4B). Co-expression of $\sigma \mathrm{NS}$ $\mathrm{N}-\mathrm{MYC}$ with either $\mu 2-\mathrm{C}-\mathrm{HA}$ or $\lambda 1-\mathrm{N}-\mathrm{HA}$, i.e. in the absence of $\mu \mathrm{NS}$, did not alter staining patterns, and the viral factory-like structures were not formed (not shown).

\section{$\sigma \mathrm{NS}$ and $\boldsymbol{\mu} \mathbf{2}$ interact with $\boldsymbol{\mu N S}$}

Immunoprecipitation and western blotting were performed to confirm interactions between PRV $\mu \mathrm{NS}$ and each of $\sigma \mathrm{NS}, \lambda 1$ and $\mu 2$ (Figure 5). EPC cells were cotransfected with $\mu \mathrm{NS}-\mathrm{N}-\mathrm{FLAG}$ and separately with the $\sigma \mathrm{NS}-\mathrm{N}-\mathrm{MYC}, \lambda 1-\mathrm{N}-\mathrm{HA}$ and $\mu 2-\mathrm{C}-\mathrm{HA}$ constructs. The results confirmed that $\mu \mathrm{NS}$ interacts with $\sigma \mathrm{NS}$ and $\mu 2$. Interaction with $\lambda 1$ on the other hand (Figure 5), or to the negative control ISAV-S8ORF2 protein, was not observed (not shown). 

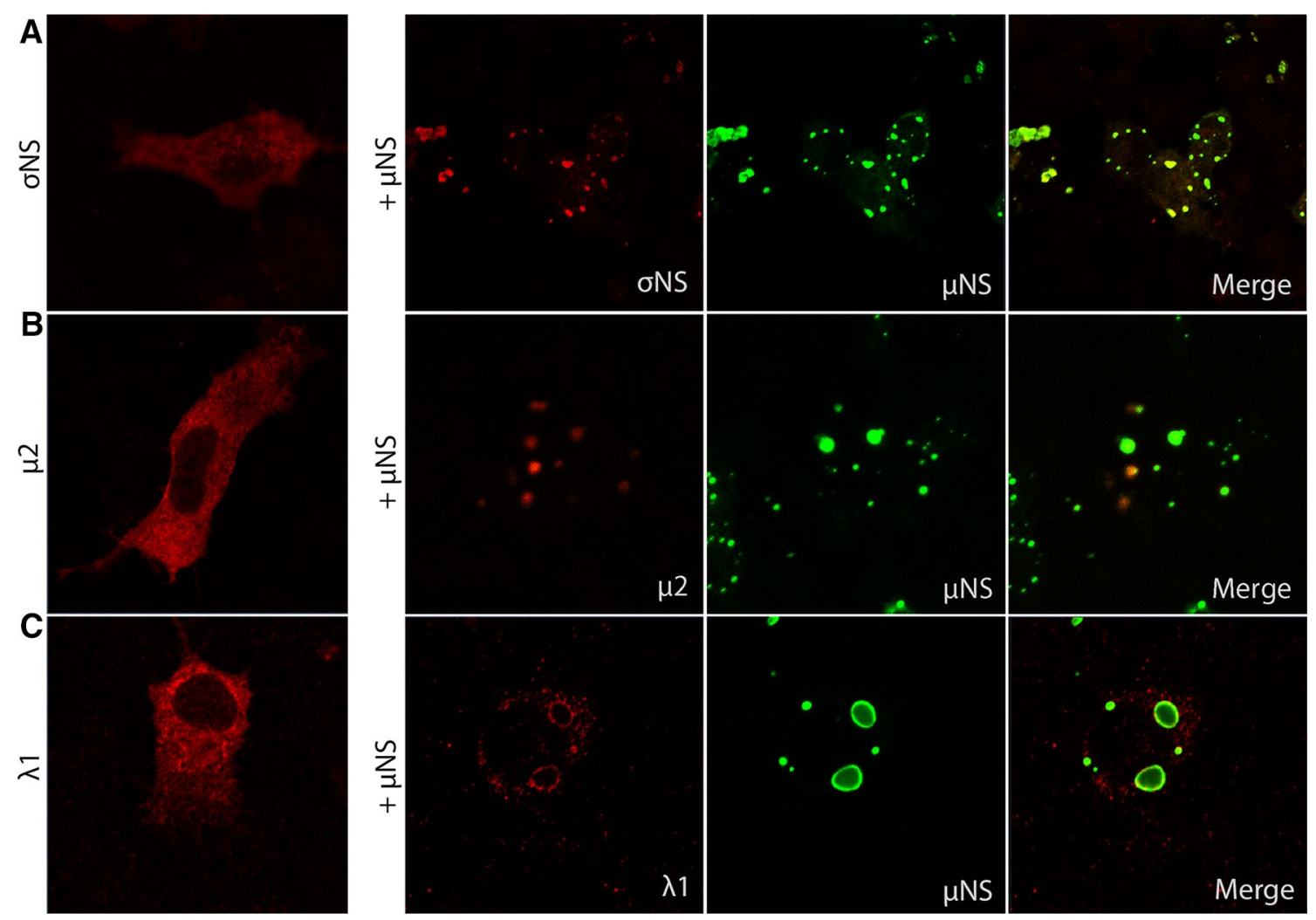

Figure 4 Co-transfections with $\boldsymbol{\mu N S}$. EPC cells transfected with constructs encoding $\sigma N S, \mu 2$ and $\lambda 1$ and co-transfected with $\mu N S$. The cells were processed for confocal microscopy $48 \mathrm{hpt}$. A EPC cells transfected with $\sigma \mathrm{NS}$ alone and cotransfected with $\mu \mathrm{NS}$. B EPC cells transfected with $\mu 2$ alone and cotransfected with $\mu N S$. C EPC cells transfected with $\lambda 1$ alone and cotransfected with $\mu N S$.

\section{Truncated $\mu$ NS proteins}

EPC cells were transfected with plasmid constructs encoding the truncated $\mu \mathrm{NS}$ variants $\mu \mathrm{NS}-\Delta 743-752, \mu \mathrm{NS}$ $\Delta 736-752, \mu N S-\Delta 1-401$ and $\mu$ NS- $\Delta 402-752$ (Figure 1 ). Small, factory-like globular inclusions were formed by $\mu \mathrm{NS} \Delta 743-752$ and $\mu \mathrm{NS} \Delta 736-752$ (Figure $6 \mathrm{~A}$ and B). Individual co-expression of these $\mu \mathrm{NS}$ truncated variants with $\sigma \mathrm{NS}-\mathrm{N}-\mathrm{MYC}$ recruited the latter protein to the factorylike inclusions, similar to that observed with full-length $\mu \mathrm{NS}$ (Figures $4 \mathrm{~A}, 6 \mathrm{~A}$ and $\mathrm{B}$ ). The $\mu \mathrm{NS} \Delta 1-401$ protein formed small dense irregular or granular structures in the cytoplasm with reminiscences to the globular structures formed by the full-length protein (Figure $6 \mathrm{C}$ ). The $\mu \mathrm{NS} \Delta 1$ 401 truncated version did also recruit and change the distribution pattern of $\sigma \mathrm{NS}$ (Figures $3 \mathrm{~B}$ and $6 \mathrm{C}$ ). In contrast, $\mu \mathrm{NS} \Delta 402-752$ was evenly distributed in the cytoplasm, and did not form viral factory-like structures. When $\mu \mathrm{NS} \Delta 402$ 752 was expressed together with $\sigma \mathrm{NS}$, both proteins were evenly dispersed throughout the cytoplasm (Figure 6D).

\section{Discussion}

The reoviral factories are the sites for virus replication and particle assembly [19]. The MRV $\mu$ NS is the scaffolding protein organizing the viral factories including gathering of core proteins, while the $\sigma \mathrm{NS}$ protein facilitates construction of core particles and subsequent particle assembly $[20,24,29,34]$. Viral factory-like structures have been observed in PRV infected Atlantic salmon erythrocytes in both in vivo and ex vivo experiments $[22,23]$. In this study we demonstrated that PRV $\mu$ NS alone forms dense globular, viral factory-like cytoplasmic inclusions. The globular, cytoplasmic distribution of $\mu$ NS was not seen for the non-structural $\sigma \mathrm{NS}$ or the structural $\mu 2$ and $\lambda 1$ PRV proteins. However, these proteins changed their distribution pattern and co-localized with $\mu \mathrm{NS}$ in the dense globular structures when they were co-transfected with $\mu \mathrm{NS}$. Co-transfection of $\sigma \mathrm{NS}$ with $\mu 2$ or $\lambda 1$ did not cause changes in distribution pattern. Expression of the $\mathrm{N}$-terminal 401 amino acids did not form viral factorylike structures, mapping this feature to the remaining C-terminal 351 amino acids. Immunoprecipitation and subsequent Western blot analysis confirmed the association between $\mu \mathrm{NS}-\sigma \mathrm{NS}$ and $\mu \mathrm{NS}-\mu 2$. Our findings strongly suggests that $\mu \mathrm{NS}$ is the prime organizer of viral factories for PRV. 


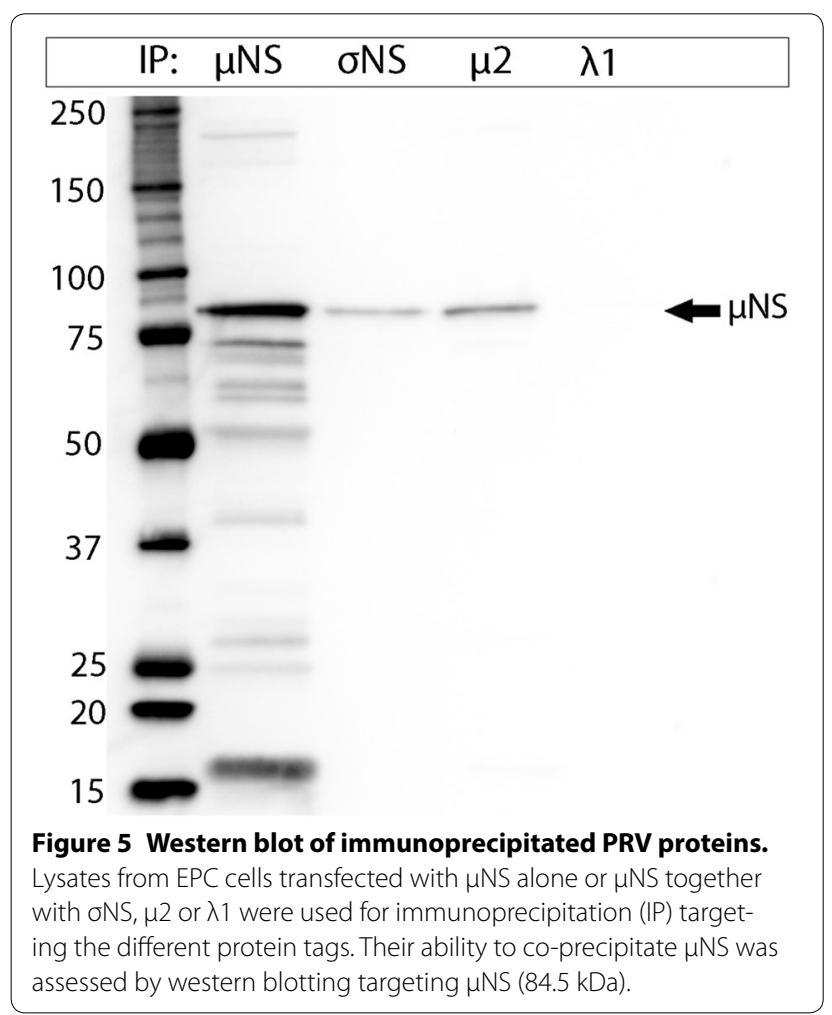

MRV strains exhibit differences in viral inclusion morphology. Reovirus type 1 Lang (T1L) forms filamentous inclusions, whereas type 3 Dearing (T3D) forms punctate or globular inclusions $[20,35]$. These morphologic differences are determined by the ability of the virus to interact with the microtubule system, a feature mapped to MRV $\mu 2$ [35]. In the filamentous factories, $\mu 2$ co-localize with and stabilize microtubules when expressed in cells in the absence of other viral proteins [20,35]. PRV inclusions appear similar to the globular inclusion type, closely resembling the $\mu \mathrm{NS}$-containing globular viral factories in reovirus T3D infected cells [35]. We cannot exclude that there are strains of PRV that forms filamentous inclusions. There might be several not yet recognized PRVlike viruses that infect other salmonid fish species. It has been proposed that the larger surface area of filamentous inclusions allow for more efficient viral replication through better access to small-molecule substrates or newly synthesized proteins from the surrounding cytosol [35]. Immunofluorescence and confocal microscopy have been used to identify globular and filamentous inclusions after transfection with expression plasmids encoding proteins from MRV and ARV [27, 36, 37].

Viral factories commonly form early in reovirus infection as small punctate structures throughout the cytoplasm that increase in size and become more perinuclear during infection [20]. The factories recruit viral proteins, which allow the efficient assembly of virus core particles $[34,38]$. We observed that PRV $\mu$ NS guided the $\sigma \mathrm{NS}, \mu 2$ and $\lambda 1$ proteins to the viral factories. Our rationale for choosing $\sigma \mathrm{NS}, \mu 2$ and $\lambda 1$ as co-transfectants was that these are examples of non-structural $(\sigma N S)$ and structural ( $\mu 2$ and $\lambda 1$ ) proteins in the core particle. MRV $\mu$ NS and $\sigma \mathrm{NS}$ are found in the first detectable viral proteinRNA complexes in MRV infected cells and form cytoplasmic inclusions similar to the viral factory-like structures formed in the absence of viral infection [36]. Analysis of MRV $\mu$ NS transfected cells revealed that at $6 \mathrm{hpt}, \mu \mathrm{NS}$ inclusions were uniformly small and spread throughout the cytoplasm, whereas at $18 \mathrm{hpt}$ and $36 \mathrm{hpt}$, larger perinuclear inclusions were present along with smaller inclusions [20]. In addition to its association with $\sigma \mathrm{NS}$, MRV $\mu$ NS has been shown to interact with each of the five structural proteins that make up the core particle $(\lambda 1$, $\lambda 2, \lambda 3, \sigma 2$ and $\mu 2)[24,34]$. Although it generally occurs within $18 \mathrm{hpt}$, strong co-localization between MRV $\mu \mathrm{NS}$ and the core surface proteins have been observed as soon as $6 \mathrm{~h}$ post infection [34]. Since PRV replicates at lower temperatures than MRV, the process of assembling core proteins to viral factories occurs at a slower rate. Studies on the ARV have identified a similar role of $\mu \mathrm{NS}$ in forming viral factories [27].

The nature of the globular inclusions and their interactions with other PRV proteins might differ in erythrocytes and established cell lines. However, neither cell line nor $\mathrm{C}$ - or $\mathrm{N}$-terminal epitope tagging influenced the formation of dense globular structures by the PRV $\mu$ NS. Transfection of salmon erythrocytes was not successful (data not shown). Still, globular-type inclusions are common in naturally PRV infected erythrocytes. This indicates that the formation of globular inclusion structures is an intrinsic property of $\mu \mathrm{NS}$.

The ability of $\mu$ NS to redirect the subcellular localizations of other PRV proteins can be mediated through protein-protein interactions. This was observed for $\sigma \mathrm{NS}$ and $\mu 2$ following immunoprecipitation and western blotting. Many cellular proteins are only functional when localized to specific cellular compartments, and translocation to the appropriate sites can serve to regulate protein function [36]. Reovirus proteins involved in replication are only active within functional centers characterized by a particular location and protein composition [36]. We could not demonstrate protein-protein interaction between $\mu \mathrm{NS}$ and $\lambda 1$, although confocal imaging clearly proved redistribution of $\lambda 1$ when the protein was co-expressed with $\mu \mathrm{NS}$. Interaction(s) between $\mu \mathrm{NS}$ and $\lambda 1$ is therefore likely but perhaps through the involvement of a third cellular protein. Alternatively, the binding affinities between the two proteins are below the threshold detectable by the conditions used in the 


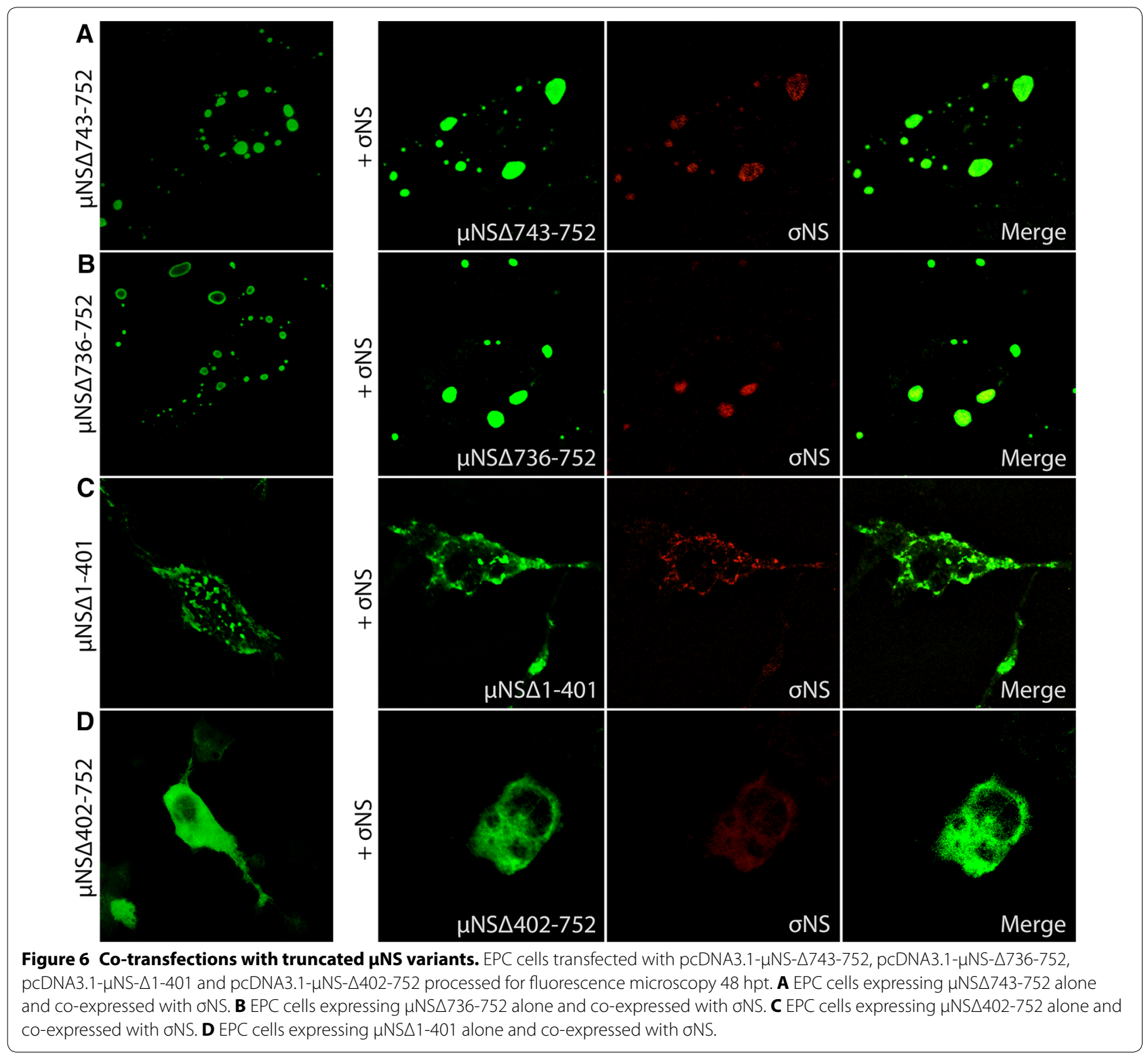

immunoprecipitation- and western blot assays. Further investigations are needed to study the mechanisms involved in $\lambda 1$ redistribution when co-expressed with $\mu N S$. Since $\mu$ NS expressed alone forms viral factory-like inclusions, and is responsible for the redistribution of other PRV proteins, it is likely one of the first proteins involved in virus factory formation and thereby essential in the early steps of viral replication.

Staining of $\sigma \mathrm{NS}$, and to some extend $\mu 2$, was observed in the nucleus of transfected cells. The size of the $\sigma \mathrm{NS}$ protein, predicted to be $39.1 \mathrm{kDa}$, may allow passive diffusion through the nuclear pores, whereas the $86 \mathrm{kDa} \mu 2$ protein exceeds the $40 \mathrm{kDa}$ limit for passive diffusion [39]. MRV $\sigma \mathrm{NS}$ and $\mu 2$ are both shown to be distributed in the nucleus and the cytoplasm of transfected and infected cells. The ability of MRV $\sigma \mathrm{NS}$ to locate in the nucleus of infected cells has been linked to its nucleic acid binding capability, while the presence of MRV $\mu 2$ in the nucleus of transfected cells is explained by predicted nuclear import and export signals [20, 24, 40-42]. There are no predicted classical nuclear localization signals (NLSs) in PRV $\sigma$ NS [8] or PRV $\mu 2$ (present study, using PSORTII and NLS mapper). The presence of nuclear export signals (NES) have though been predicted for both proteins. Neither $\sigma \mathrm{NS}$ nor $\mu 2$ was found in the nucleus after co-transfection with $\mu \mathrm{NS}$. As $\mu \mathrm{NS}$ does not 
localize to the nucleus, an explanation might be that $\mu \mathrm{NS}$ sequesters $\sigma \mathrm{NS}$ and $\mu 2$ within the cytoplasmic inclusions, thus reducing the amount of free $\sigma \mathrm{NS}$ and $\mu 2$ to enter the nucleus. This has also been proposed for MRV $\sigma \mathrm{NS}$ and $\mu 2[20,40]$. Further studies are needed to excavate the functional roles of the observed nuclear localization of PRV $\sigma \mathrm{NS}$ and $\mu 2$.

The C-terminal part of MRV $\mu$ NS contains four distinct regions comprising 250 amino acids that are sufficient to form viral factories [29]. These regions include two predicted coiled-coil domains, a linker region between the coiled coils containing a putative zinc hook, and a short C-terminal tail [24]. PRV $\mu$ NS may contain a coiled-coil motif in its C-terminal region [8]. A deletion of the eight C-terminal amino acids of MRV $\mu$ NS results in diffusely distributed protein throughout the cytoplasm and the nucleus, suggesting that these amino acids are necessary for inclusion formation [29]. PRV $\mu$ NS also contains a high $\alpha$-helical content in its C-terminal region although the sequence identity to the homologous MRV protein is low [8]. In fact, the predicted secondary structure profiles of MRV and PRV $\mu$ NS show significant similarities, highlighting the importance of conserving structural features over primary sequence for the function of homologues proteins across evolutionary lines. Still, the two C-terminally truncated forms of $\mu \mathrm{NS}$ containing deletions of 10 and 17 amino acids, respectively, formed viral factory-like structures when expressed in EPC cells, indicating that factory formation is not dependent on these amino acids. Deletion of the $401 \mathrm{~N}$-terminal amino acids seemed to have some influence on the viral factory formation, but the protein still accumulated in granular structures and retained its ability to recruit $\sigma \mathrm{NS}$. Deletions of the 351 C-terminal amino acids, on the other hand, resulted in diffusely distributed protein and absence of globular inclusions. This indicates that the $\mathrm{C}$-terminal region of $\mu \mathrm{NS}$ is essential for factory formation. The $\mathrm{N}$-terminal region of PRV $\mu$ NS displays a somewhat higher level of secondary structure conservation when compared to MRV. In MRV, this region of $\mu \mathrm{NS}$ is crucial for interactions with $\sigma \mathrm{NS}, \mu 2, \lambda 1$ and $\lambda 2[34,38]$.

In conclusion, our results strongly suggest that PRV $\mu N S$ protein is essential for factory formation and assembly of viral proteins, similar to that of $\mu \mathrm{NS}$ of other orthoreoviruses. Further studies on both the structural and functional properties of PRV proteins can provide important information relating to disease development following PRV infections.

\section{Competing interests}

The authors declare that they have no competing interests.

\section{Authors' contributions}

$\mathrm{HH}$ constructed the expression plasmids, performed the transfections, the immunofluorescence microscopy, the immunoprecipitation and western blot analyses and drafted the manuscript. IN participated in the construction of expression plasmids and in the immunoprecipitation and western blot analyses. TM carried out the computer analyses and participated in the construction of truncated proteins. $\varnothing \mathrm{W}$ participated in the construction of expression plasmids and in the design of the study. MD and ER conceived of the study, and participated in its design and coordination and helped to draft the manuscript. All authors read and approved the final manuscript.

\section{Acknowledgements}

The Research Council of Norway supported the research with grant \#237315/ E40 and \#235788. We would also like to thank Stine Braaen and Even Thoen for technical and scientific assistance in the project.

\section{Author details}

${ }^{1}$ Department of Food Safety and Infectious Biology, Faculty of Veterinary Medicine and Biosciences, Norwegian University of Life Sciences, Postboks 8146 Dep, 0033 Oslo, Norway. ${ }^{2}$ Department of Parasitology, Norwegian Veterinary Institute, Postboks 750 Sentrum, 0106 Oslo, Norway. ${ }^{3}$ Department of Immunology, Norwegian Veterinary Institute, Postboks 750 Sentrum, 0106 Oslo, Norway.

Received: 30 January 2015 Accepted: 4 September 2015

Published online: 08 January 2016

\section{References}

1. Palacios G, Løvoll M, Tengs T, Hornig M, Hutchison S, Hui J, Kongtorp RT, Savji N, Bussetti AV, Solovyov A, Kristoffersen AB, Celeone C, Street C, Trifonov V, Hirschberg DL, Rabadan R, Egholm M, Rimstad E, Lipkin WI (2010) Heart and skeletal muscle inflammation of farmed salmon is associated with infection with a novel reovirus. PLoS One 5:e11487

2. Finstad $\varnothing W$, Falk K, Løvoll M, Evensen $\varnothing$, Rimstad E (2012) Immunohistochemical detection of piscine reovirus (PRV) in hearts of Atlantic salmon coincide with the course of heart and skeletal muscle inflammation (HSMI). Vet Res 43:27

3. Kongtorp RT, Halse M, Taksdal T, Falk K (2006) Longitudinal study of a natural outbreak of heart and skeletal muscle inflammation in Atlantic salmon, Salmo salar L. J Fish Dis 29:233-244

4. Løvoll M, Alarcon M, Bang Jensen B, Taksdal T, Kristoffersen AB, Tengs $T$ (2012) Quantification of piscine reovirus (PRV) at different stages of Atlantic salmon Salmo salar production. Dis Aquat Organ 99:7-12

5. Rimstad E (2011) Examples of emerging virus diseases in salmonid aquaculture. Aquac Res 42:86-89

6. Garseth AH, Fritsvold C, Opheim M, Skjerve E, Biering E (2013) Piscine reovirus (PRV) in wild Atlantic salmon, Salmo salar L., and sea-trout, Salmo trutta L., in Norway. J Fish Dis 36:483-493

7. Nibert ML, Duncan R (2013) Bioinformatics of recent aqua- and orthoreovirus isolates from fish: evolutionary gain or loss of FAST and fiber proteins and taxonomic implications. PLoS One 8:e68607

8. Markussen T, Dahle MK, Tengs T, Løvoll M, Finstad ØW, Wiik-Nielsen CR, Grove S, Lauksund S, Robertsen B, Rimstad E (2013) Sequence analysis of the genome of piscine orthoreovirus (PRV) associated with heart and skeletal muscle inflammation (HSMI) in Atlantic salmon (Salmo salar). PLoS One 8:e70075

9. Kibenge MJ, Iwamoto T, Wang Y, Morton A, Godoy MG, Kibenge FS (2013) Whole-genome analysis of piscine reovirus (PRV) shows PRV represents a new genus in family reoviridae and its genome segment $S 1$ sequences group it into two separate sub-genotypes. Virol J 10:230

10. Wessel $\varnothing$, Nyman IB, Markussen T, Dahle MK, Rimstad E (2015) Piscine orthoreovirus (PRV) o3 protein binds dsRNA. Virus Res 198:22-29

11. Key T, Read J, Nibert ML, Duncan R (2013) Piscine reovirus encodes a cytotoxic, non-fusogenic, integral membrane protein and previously unrecognized virion outer-capsid proteins. J Gen Virol 94:1039-1050

12. Guglielmi KM, Johnson EM, Stehle T, Dermody TS (2006) Attachment and cell entry of mammalian orthoreovirus. Curr Top Microbiol 309:1-38

13. Novoa RR, Calderita G, Arranz R, Fontana J, Granzow H, Risco C (2005) Virus factories: associations of cell organelles for viral replication and morphogenesis. Biol Cell 97:147-172 
14. Becker MM, Peters TR, Dermody TS (2003) Reovirus ơNS and $\mu N S$ proteins form cytoplasmic inclusion structures in the absence of viral infection. J Virol 77:5948-5963

15. Schiff LA, Nibert ML, Tyler KL (2007) Orthoreoviruses and their replication. In: Knipe DM, Howley PM, Fields BN (eds) Fields virology, vol 2, 5th edn. Wolters Kluwer/Lippincott Williams \& Wilkins, Philadelphia

16. Paul D, Bartenschlager R (2013) Architecture and biogenesis of plusstrand RNA virus replication factories. World J Virol 2:32-48

17. Netherton C, Moffat K, Brooks E, Wileman T (2007) A guide to viral inclusions, membrane rearrangements, factories, and viroplasm produced during virus replication. Adv Virus Res 70:101-182

18. de Castro IF, Volonte L, Risco C (2013) Virus factories: biogenesis and structural design. Cell Microbiol 15:24-34

19. Fernandez de Castro I, Zamora PF, Ooms L, Fernandez JJ, Lai CM, Mainou BA, Dermody TS, Risco C (2014) Reovirus forms neo-organelles for progeny particle assembly within reorganized cell membranes. MBio 5:e00931-e01013

20. Broering TJ, Parker JS, Joyce PL, Kim J, Nibert ML (2002) Mammalian reovirus nonstructural protein microNS forms large inclusions and colocalizes with reovirus microtubule-associated protein micro2 in transfected cells. J Virol 76:8285-8297

21. Schmid M, Speiseder T, Dobner T, Gonzalez RA (2014) DNA virus replication compartments. J Virol 88:1404-1420

22. Finstad $\varnothing W$, Dahle MK, Lindholm TH, Nyman IB, Løvoll M, Wallace C, Olsen CM, Storset AK, Rimstad E (2014) Piscine orthoreovirus (PRV) infects Atlantic salmon erythrocytes. Vet Res 45:35

23. Wessel $\varnothing$, Olsen CM, Rimstad E, Dahle MK (2015) Piscine orthoreovirus (PRV) replicates in Atlantic salmon (Salmo Salar L.) erythrocytes ex vivo. Vet Res 46:26

24. Miller CL, Arnold MM, Broering TJ, Hastings CE, Nibert ML (2010) Localization of mammalian orthoreovirus proteins to cytoplasmic factory-like structures via nonoverlapping regions of microNS. J Virol 84:867-882

25. Ivanovic T, Boulant S, Ehrlich M, Demidenko AA, Arnold MM, Kirchhausen T, Nibert ML (2011) Recruitment of cellular clathrin to viral factories and disruption of clathrin-dependent trafficking. Traffic 12:1179-1195

26. McCutcheon AM, Broering TJ, Nibert ML (1999) Mammalian reovirus M3 gene sequences and conservation of coiled-coil motifs near the carboxyl terminus of the microNS protein. Virology 264:16-24

27. Touris-Otero F, Cortez-San Martin M, Martinez-Costas J, Benavente J (2004) Avian reovirus morphogenesis occurs within viral factories and begins with the selective recruitment of sigmaNS and lambdaA to microNS inclusions. J Mol Biol 341:361-374

28. Brandariz-Nunez A, Menaya-Vargas R, Benavente J, Martinez-Costas J (2010) Avian reovirus microNS protein forms homo-oligomeric inclusions in a microtubule-independent fashion, which involves specific regions of its C-terminal domain. J Virol 84:4289-4301

29. Broering TJ, Arnold MM, Miller CL, Hurt JA, Joyce PL, Nibert ML (2005) Carboxyl-proximal regions of reovirus nonstructural protein muNS necessary and sufficient for forming factory-like inclusions. J Virol 79:6194-6206
30. Busch LK, Rodriguez-Grille J, Casal Jl, Martinez-Costas J, Benavente J (2011) Avian and mammalian reoviruses use different molecular mechanisms to synthesize their microNS isoforms. J Gen Virol 92:2566-2574

31. Yachdav G, Kloppmann E, Kajan L, Hecht M, Goldberg T, Hamp T, Honigschmid P, Schafferhans A, Roos M, Bernhofer M, Richter L, Ashkenazy H, Punta M, Schlessinger A, Bromberg Y, Schneider R, Vriend G, Sander C, Ben-Tal N, Rost B (2014) PredictProtein-an open resource for online prediction of protein structural and functional features. Nucl Acids Res 42:W337-W343

32. Terpe K (2003) Overview of tag protein fusions: from molecular and biochemical fundamentals to commercial systems. Appl Microbiol Biotechnol 60:523-533

33. Garcia-Rosado E, Markussen T, Kileng O, Baekkevold ES, Robertsen B, Mjaaland S, Rimstad E (2008) Molecular and functional characterization of two infectious salmon anaemia virus (ISAV) proteins with type I interferon antagonizing activity. Virus Res 133:228-238

34. Broering TJ, Kim J, Miller CL, Piggott CD, Dinoso JB, Nibert ML, Parker JS (2004) Reovirus nonstructural protein muNS recruits viral core surface proteins and entering core particles to factory-like inclusions. J Virol 78:1882-1892

35. Parker JS, Broering TJ, Kim J, Higgins DE, Nibert ML (2002) Reovirus core protein mu2 determines the filamentous morphology of viral inclusion bodies by interacting with and stabilizing microtubules. J Virol 76:4483-4496

36. Becker MM, Peters TR, Dermody TS (2003) Reovirus sigmaNS and muNS proteins form cytoplasmic inclusion structures in the absence of viral infection. J Virol 77:5948-5963

37. Becker MM, Goral MI, Hazelton PR, Baer GS, Rodgers SE, Brown EG, Coombs KM, Dermody TS (2001) Reovirus sigmaNS protein is required for nucleation of viral assembly complexes and formation of viral inclusions. J Virol 75:1459-1475

38. Carroll K, Hastings C, Miller CL (2014) Amino acids 78 and 79 of mammalian orthoreovirus protein microNS are necessary for stress granule localization, core protein lambda2 interaction, and de novo virus replication. Virology 448:133-145

39. Mohr D, Frey S, Fischer T, Guttler T, Gorlich D (2009) Characterisation of the passive permeability barrier of nuclear pore complexes. EMBO J 28:2541-2553

40. Gillian AL, Nibert ML (1998) Amino terminus of reovirus nonstructural protein sigmaNS is important for ssRNA binding and nucleoprotein complex formation. Virology 240:1-11

41. Kobayashi T, Ooms LS, Chappell JD, Dermody TS (2009) Identification of functional domains in reovirus replication proteins muNS and mu2. J Virol 83:2892-2906

42. Ooms LS, Kobayashi T, Dermody TS, Chappell JD (2010) A post-entry step in the mammalian orthoreovirus replication cycle is a determinant of cell tropism. J Biol Chem 285:41604-41613

\section{Submit your next manuscript to BioMed Central and we will help you at every step:}

- We accept pre-submission inquiries

- Our selector tool helps you to find the most relevant journal

- We provide round the clock customer support

- Convenient online submission

- Thorough peer review

- Inclusion in PubMed and all major indexing services

- Maximum visibility for your research

Submit your manuscript at www.biomedcentral.com/submit

\section{() Biomed Central}

